\title{
Porosification of Surface of Platinum Electrode by Anisotropic Etching
}

\author{
Yasuo Terasawa, ${ }^{1,2 *}$ Hiroyuki Tashiro, ${ }^{3}$ Yukari Nakano, ${ }^{1,2}$ and Jun Ohta ${ }^{2}$ \\ ${ }^{1}$ Artificial Vision Institute, R\&D Division, Nidek Co., Ltd., 13-2 Hama-cho, Gamagori, Aichi 443-0036, Japan \\ ${ }^{2}$ Materials Science, Nara Institute of Science and Technology, Takayama-cho, Ikoma, Nara 630-0192, Japan \\ ${ }^{3}$ Department of Health Sciences, Kyushu University, 3-1-1 Maidashi, Higashi-ku, Fukuoka 812-8582, Japan
}

(Received February 19, 2019; accepted April 10, 2019)

Keywords: neural prostheses, electrode, porosification, anisotropic etching

In neural prosthetics, stimulation electrodes are one of the most important components for stimulating neural tissue. It is known that the surface roughening of stimulation electrodes can improve their performance. In this study, we describe a method of producing platinum electrodes with a porous surface by anisotropic etching. Anisotropic etching was achieved by applying a DC voltage to a platinum electrode in physiological saline. The charge injection capacity $Q_{i n j}$ almost doubled after etching, compared with that before etching. Furthermore, continuous current pulsing to the porous electrode in phosphate-buffered physiological saline for four months did not show any apparent deterioration in surface morphology or electrochemical properties, suggesting that the proposed porosification method improves the performance of stimulation electrodes.

\section{Introduction}

In neural prosthetics, electrical stimulation is widely used and contributes to improving the quality of life for many patients. There are many commercial neural stimulators, which include the following: cardiac pacemakers that electrically stimulate the cardiac muscle to treat arrhythmias and heart failure; deep brain stimulator to improve Parkinson's disease or essential tremor; functional electrical stimulator to stimulate peripheral nerve and move a paralyzed muscle; and cochlear implants that transmit auditory information to the hearing impaired. ${ }^{(1)}$ In recent years, visual prostheses have also been put into practical use ${ }^{(2,3)}$ and have been reported to partially restore vision in blind patients.

The electrodes used for electrical stimulation are required to deliver safe levels of therapeutic stimulation resulting in the excitation of the target nerve. The maximum injectable charge per unit area that can be delivered without an accompanying irreversible electrochemical reaction, such as the electrolysis of water, is known as the charge injection capacity $\left(Q_{i n j}\right) .{ }^{(4,5)}$ A large $Q_{i n j}$ is an indicator of the performance of a stimulation electrode; hence, a large $Q_{i n j}$ is a desirable characteristic. Additionally, in some applications of neural prostheses, it is essential

*Corresponding author: e-mail: yasuo_terasawa@nidek.co.jp

https://doi.org/10.18494/SAM.2019.2334 
that the impedance of the stimulation electrode is sufficiently small. If the impedance is large, necessary charge injection cannot be performed owing to the driving capability limitation of the driving circuit. Furthermore, high impedance leads to heat generation, which could damage the neural tissue.

One solution to reduce impedance would be to increase the geometrical dimensions of the stimulation electrode such as diameter and height. However, as the size of the electrode increases, the localized delivery of electrical stimulation becomes more difficult, resulting in the diffusion of the charge delivered to biological tissues. Such precise localization is particularly important in artificial sensory organs such as cochlear implants and visual prostheses because they need a relatively large number of electrodes to send acoustic or visual information to patients. For this reason, depending on their applications, stimulation electrodes are required to exhibit high $Q_{i n j}$ and low impedance, while satisfying the constraints of shape and size for their specific applications.

For electrodes to exhibit high $Q_{i n j}$, electrodes have conventionally been coated with highperformance materials such as titanium nitride with a large effective surface area ${ }^{(6)}$ and iridium oxide $^{(7,8)}$ formed by sputtering or electrochemical activation. Recently, a conductive polymer, poly-(3,4-ethylenedioxythiophene) $\left(\right.$ PEDOT) ${ }^{(9,10)}$ and low-impedance platinum iridium ${ }^{(11)}$ have also been proposed. Surface coatings such as these are useful in improving the electrode performance, but problems, such as the delamination of the coating film, have been reported and are dependent on the parameters of the applied current. ${ }^{(12)}$ Another solution to increase $Q_{i n j}$ would be to modify the shape of the electrode surface. The introduction of dips, kinks, holes, and gorges on the surface results in an electrode with a much greater real surface area than its geometric surface area (GSA). Increasing the real surface area would decrease the impedance of the electrode and the charge per unit area that can be safely delivered would increase. Surface roughening processes, such as laser irradiation, ${ }^{(13)}$ electrochemical etching, ${ }^{(14)}$ and electrochemical platinum deposition, ${ }^{(15)}$ are well known for increasing the real surface area. Here, we report a novel technique involving the anisotropic etching of the surface of a bulk platinum electrode to increase its real surface area. Since this method employs a dissolution phenomenon, problems observed with thin-film charge injection coating techniques, such as the delamination of the film and the mechanical damage of the deposited film, are not likely to occur; therefore, highly reliable electrodes can be obtained. Here, the anisotropic etching method is described, the resulting surface morphology and internal structure of the etched electrode are shown, and both short-term and long-term electrochemical characteristics are evaluated. Part of this research was presented at an international meeting. ${ }^{(16)}$

\section{Materials and Methods}

\subsection{Fabrication}

\subsubsection{Electrodes}

Methods for making electrodes and electrode substrates have been described. ${ }^{(17)}$ The electrode used here was developed for a suprachoroidal retinal prosthesis. ${ }^{(18)}$ Briefly, a bullet- 
shaped stimulation electrode was produced by mechanically machining a 1-mm-diameter platinum rod. The diameter and height of the resulting electrode were both $0.5 \mathrm{~mm}$. The tip had a hemispherical shape (Fig. 1) and the geometric surface area of the exposed part was 0.007854 $\mathrm{cm}^{2}$. The bottom of the bullet-shaped electrode was embedded in a parylene substrate for insulation.

\subsubsection{Anisotropic etching}

The bullet-shaped electrode and a large platinum plate $\left(>1 \mathrm{~cm}^{2}\right)$, which was used as a counter electrode, were immersed in $0.01 \mathrm{~mol} / \mathrm{L}$ phosphate-buffered saline (PBS) (P4417, Sigma-Aldrich, St. Louis, USA) at room temperature. Both the bullet-shaped electrode and the counter electrode were connected to a function generator (WF 1943, NF Corp., Yokohama, Japan). A DC voltage of $+5 \mathrm{~V}$ was applied between the bullet-shaped electrode and the counter electrode for $10 \mathrm{~s}$, and then the polarity was reversed, i.e., a DC voltage of $+5 \mathrm{~V}$ was applied between the electrodes for $10 \mathrm{~s}$. This process was repeated for $15 \mathrm{~h}$. During this process, the PBS was stirred with a magnetic stirrer to remove bubbles that had developed on the electrode surface caused by the electrolysis of water.

\subsubsection{Surface and cross-sectional observation}

The surface of the electrode after etching was observed using a scanning electron microscope (S-2600N, Hitachi High-Technologies, Tokyo, Japan). To observe the cross section of the stimulation electrode, the electrode was first embedded in epoxy resin (G-2, JEOL Ltd., Tokyo, Japan) and the side of the sample was roughly polished with sandpaper. The exposed face was polished with a cross-sectional polisher (SM-09010, JEOL Ltd., Tokyo, Japan) and the cross section of the prepared sample was observed using a scanning electron microscope (JSM5600LV, JEOL Ltd., Tokyo, Japan).

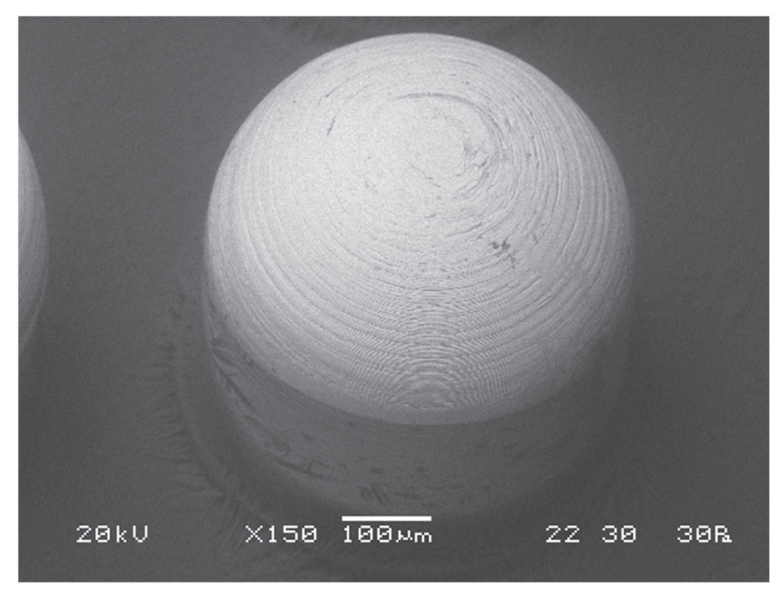

Fig. 1. Bullet-shaped electrode. This image was taken at an oblique angle of $30^{\circ}$ with respect to the vertical axis of the electrode. The backscattered electron mode of SEM was employed for imaging. 


\subsection{Long-term testing}

\subsubsection{Experimental procedure}

Three of the abovementioned bullet-shaped electrodes were used in this long-term study. Figure 2 shows the experimental procedure. First, measurements of the three bullet-shaped electrodes were performed as described in Sect. 2.2.2. Then, the electrodes were anisotropically etched as described in Sect. 2.1.2. Thereafter, the same measurements were performed and pulsating current was applied. A custom-made stimulator was used for the application of symmetric cathodic-first biphasic current pulses. The pulse width and repetition frequency were $0.5 \mathrm{~ms}$ and $50 \mathrm{~Hz}$, respectively. A pulse of $1.0 \mathrm{~mA}$ amplitude $(0.5 \mu \mathrm{C} /$ phase and 63.7 $\mu \mathrm{C} / \mathrm{cm}^{2}$ ) was used for the first week of the stimulation period and a pulse of $1.5 \mathrm{~mA}$ amplitude was used thereafter $\left(0.75 \mu \mathrm{C} /\right.$ phase and $\left.95.5 \mu \mathrm{C} / \mathrm{cm}^{2}\right)$ (Fig. 2). The change in pulse amplitude was due to the estimation of a marginal change in $Q_{i n j}$ at the end of the one week stimulation period. Electrical stimulation was carried out in $0.01 \mathrm{~mol} / \mathrm{L}$ air-equilibrated PBS. The temperature of the PBS was kept at $37^{\circ} \mathrm{C}$, using a water bath (TB-2N, AS ONE, Osaka, Japan), during the stimulation period. Measurements were conducted after 1, 2, 3, 5, and 13 weeks (Fig. 2).

\subsubsection{Measurements}

Cyclic voltammetry (CV), impedance spectroscopy, $Q_{i n j}$ measurements, and scanning electron microscopy (SEM) were performed six times as described in Fig. 2. The CV was performed in $0.01 \mathrm{~mol} / \mathrm{L}$ air-equilibrated $\mathrm{PBS}$ at room temperature using an electrochemical instrument (PGSTAT12, Metrohm Autolab, Utrecht, Netherlands) at a scanning rate of $0.05 \mathrm{~V} / \mathrm{s}$. The cathodal charge storage capacity (CSCc) was calculated by dividing the time integral value of the cathodic current of the CV by the GSA of the electrode.

An impedance spectrum was obtained using the same electrochemical instrument in the range of $10 \mathrm{~Hz}-100 \mathrm{kHz}$.

To measure $Q_{i n j}$, cathodic-first current pulses of $0.5 \mathrm{~ms}$ width were applied to the bulletshaped electrodes at room temperature in PBS. A large counter electrode (a platinum wire of

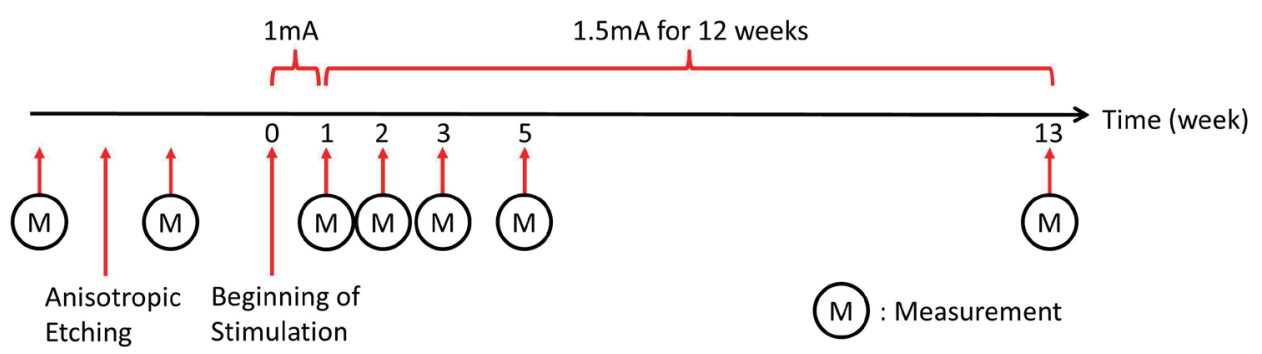

Fig. 2. (Color online) Experimental procedure. Measurements were performed before and after the anisotropic etching of the electrodes. Current pulses were then applied to the electrodes in PBS. Measurements were performed after $1,2,3,5$, and 13 weeks. 
$0.5 \mathrm{~mm}$ diameter and $50 \mathrm{~mm}$ length) was used. The electrode potential was measured using the electrometer function of the electrochemical instrument PGSTAT12 and recorded using an oscilloscope (Wavesurfer 424, Teledyne Lecroy, New York, USA). As a reference electrode in the measurement of the electrode potential, a silver/silver chloride electrode ( $\mathrm{Ag} / \mathrm{AgCl}, \mathrm{Metrohm}$ Autolab, Utrecht, Netherlands) was used. Then, $Q_{i n j}$, i.e., the maximum amount of charge per unit area that can be injected without deviating the potential window (between -0.6 and $+0.8 \mathrm{~V}$ versus $\left.\mathrm{Ag} / \mathrm{AgCl}^{(19)}\right)$, was calculated.

For the morphological examination of the electrodes, a backscattered electron image was acquired using a scanning electron microscope (S-2600N, Hitachi High-Technologies, Tokyo, Japan) in the low vacuum mode.

\subsection{Statistical analysis}

Statistical analysis was performed using commercially available software (JMP Pro 13, SAS, Cary, USA). Student's paired t-tests were performed, and where required, Bonferroni's correction was applied. In all tests, the significance level was set to $p<0.05$.

\section{Results}

\subsection{Surface and cross-sectional morphology}

\subsubsection{Surface morphology}

Figure 3 shows the images of the electrodes obtained before and after anisotropic etching. The surfaces of these two electrodes [Figs. 3(A) and 3(B)] became porous after anisotropic
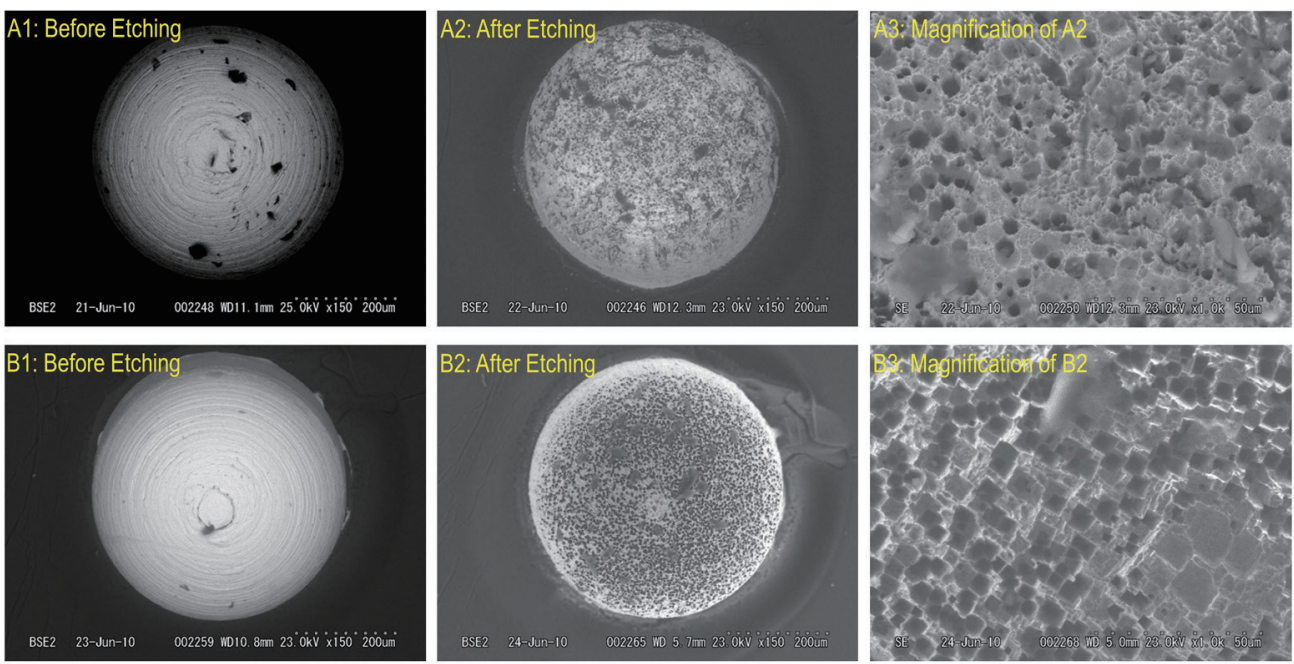

Fig. 3. (Color online) Surface morphology of two electrodes before and after anisotropic etching. Both electrodes were shown to have similar surface morphologies before etching (A1, B1), but after etching, one obtained circular pores on its surface (A2, A3) and the other had quadrangular pores (B2, B3). The backscatterd mode of SEM was employed for A1, A2, B1, and B2. The secondary electrode mode was employed for A3 and B3. 
etching, compared with those before etching. In addition, there were cases where circular holes were formed in some electrodes [Fig. 3(A3)], and rectangular holes were formed in other electrodes [Fig. 3(B3)]. The diameter of the holes was typically in the order of $10 \mu \mathrm{m}$.

\subsubsection{Cross-sectional morphology}

The cross section of an electrode after anisotropic etching is shown in Fig. 4. The crystal grains of the platinum electrode can be distinguished by electron channeling contrast imaging. Discontinuous changes in surface morphology occasionally corresponded to grain boundaries [yellow arrows in Fig. 4(B)]. The typical width and height of the crystal grain were approximately 0.5 and $5 \mu \mathrm{m}$, respectively.

\subsection{Long-term stability}

\subsubsection{Surface morphology}

No obvious deterioration in surface morphology was observed during the 13-week stimulation period in both the macroscopic images [Figs. 5(A) $-5(\mathrm{C})$ ] and the magnified views [Figs. 5(D) $-5(\mathrm{~F})]$. Note that the electrode in Fig. 5 is the same as that in Figs. 3(B1)-3(B3).

\subsubsection{Cyclic voltammogram and impedance spectrum}

Figures 6(A) and 6(C) show cyclic voltammograms and impedance spectra, respectively, measured before anisotropic etching, after etching, and during the stimulation period. Figure 6(B) shows the calculated CSCc values before anisotropic etching, after etching, and at the end of the 5th and 13th weeks of the stimulation period. Comparison before etching and after
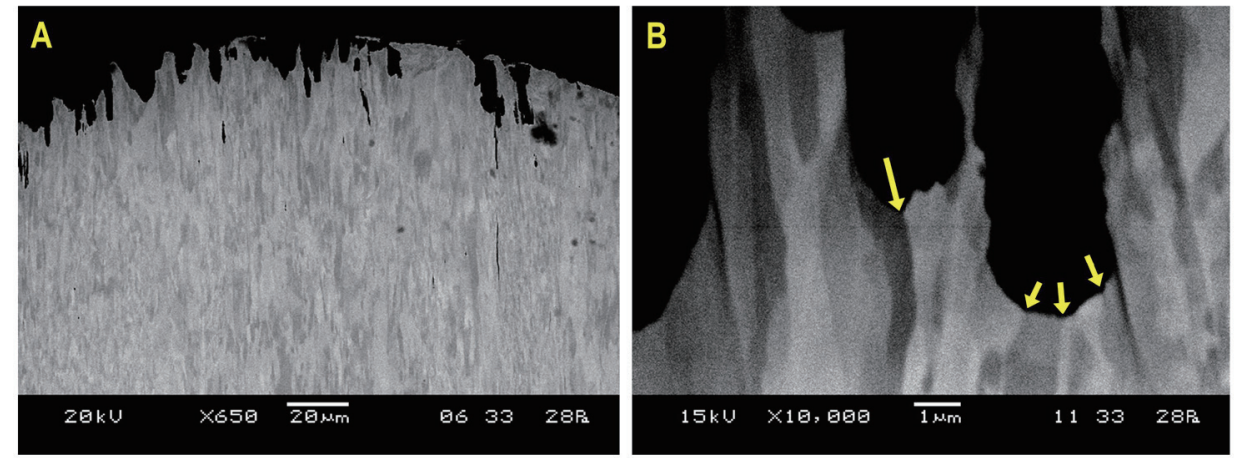

Fig. 4. (Color online) Cross section of an electrode after anisotropic etching. (A) Apex portion of the electrode. (B) Magnified view of the cross section. Discontinuous changes in surface structure occasionally corresponded to boundaries of crystal grains (yellow arrows). The backscattered mode of SEM was employed in (A) and (B). 

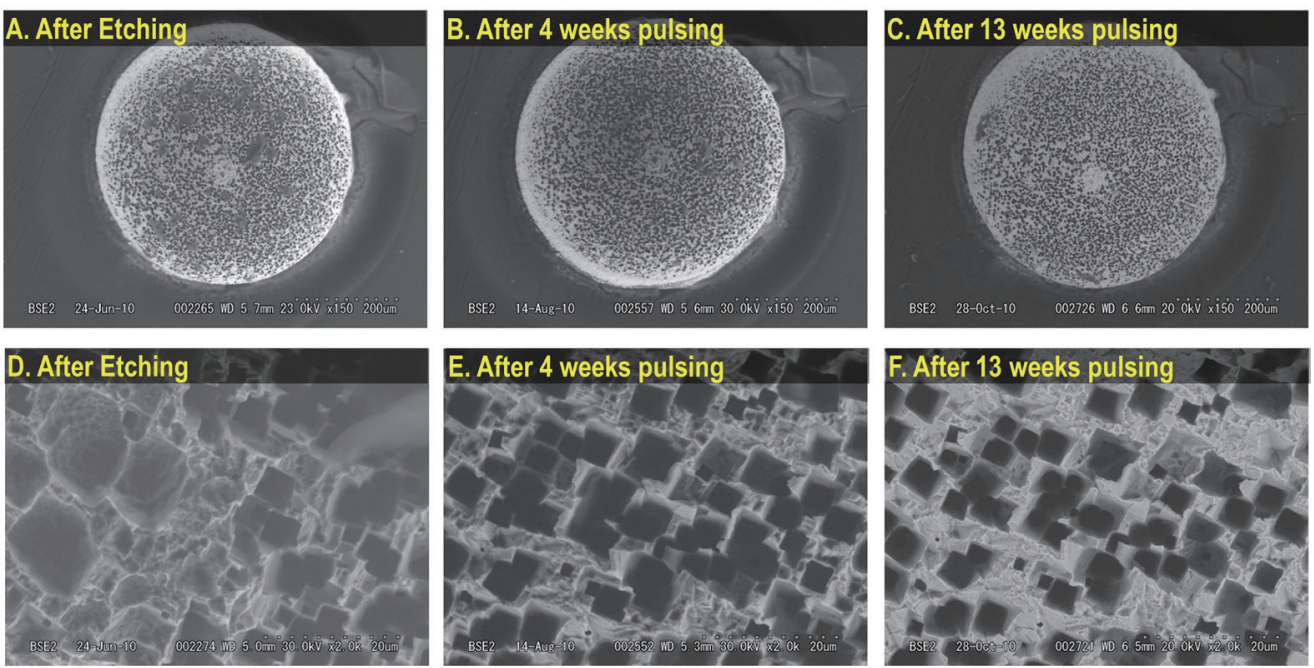

Fig. 5. (Color online) Longitudinal observation of the electrode surface over 13 weeks of electrical stimulation. Note that the observed location of the electrode surface in (D) is slightly different from that in (E) owing to unintentional shift during electron microscopy. The backscattered mode of SEM was employed for all images.
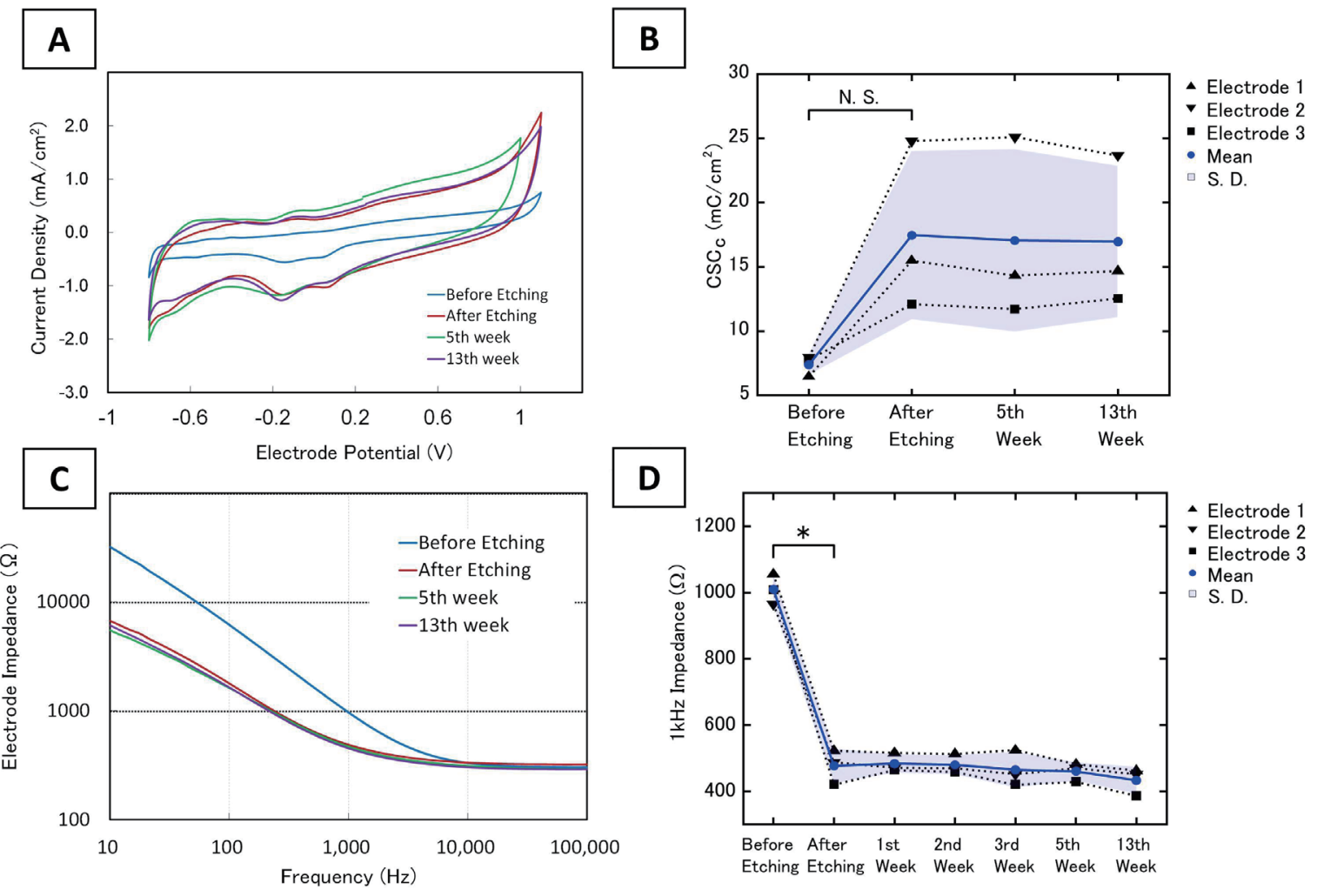

Fig. 6. (Color online) Electrochemical measurements before and after anisotropic etching, and after 5 and 13 weeks of stimulation. The graphs and histograms show (A) a cyclic voltammogram, (B) CSCc calculated from the cyclic voltammogram, (C) impedance spectra, and (D) impedance values measured at $1 \mathrm{kHz}$. In (B) and (D), the bar length and error bars represent mean and \pm S. D., respectively. The N. S. in (B) represents the lack of significance, and the asterisk * in (D) represents statistical significance $(p<0.05)$. Note that in $(A)$ and $(C)$, only representative data (corresponding to electrode no. 3) were plotted. 
etching showed that CSCc increased 2.4 times [Fig. 6(B)]; however, this increase was not statistically significant $(t=2.77, p=0.0547$, one-sided paired $t$-test). The average impedance measured at $1 \mathrm{kHz}$ significantly decreased by 50\% after anisotropic etching $(t=-16.7, p=0.0018$, one-sided paired $t$-test, Fig. 6(D). The impedance after the stimulation period at the lower frequencies was slightly smaller than that measured immediately after etching.

\subsection{3 $Q_{i n j}$}

$Q_{i n j}$ almost doubled immediately following anisotropic etching (Fig. 7). This increase was statistically significant $\left(t=3.26, p=0.0412\right.$, one-sided paired $t$-test). About $30 \%$ lower $Q_{i n j}$ was observed after the 1st and 13th weeks compared with that observed immediately after etching.

\section{Discussion}

We proposed a porosification method with anisotropic etching for platinum electrodes. The properties of the etched electrode were evaluated. The morphology of the porous surface varied depending on the electrode (Fig. 3). Since the etching conditions, such as applied voltage and solution, were the same, this difference was considered to be due to the internal structure of the stimulation electrode. Indeed, the cross-sectional observation showed a correlation between the grain boundary and the discontinuous change in surface structure (Fig. 4). However, as all the electrodes were fabricated by the same machining method, the cause of the difference in the internal structure of the electrode is unclear. In addition, the crystal grain was about $0.5 \mu \mathrm{m}$ in width and about $5 \mu \mathrm{m}$ in height (Fig. 4), whereas most of the holes formed on the electrode surface were about $10 \mu \mathrm{m}$ in diameter (Fig. 3). Furthermore, in one electrode, many rectangular

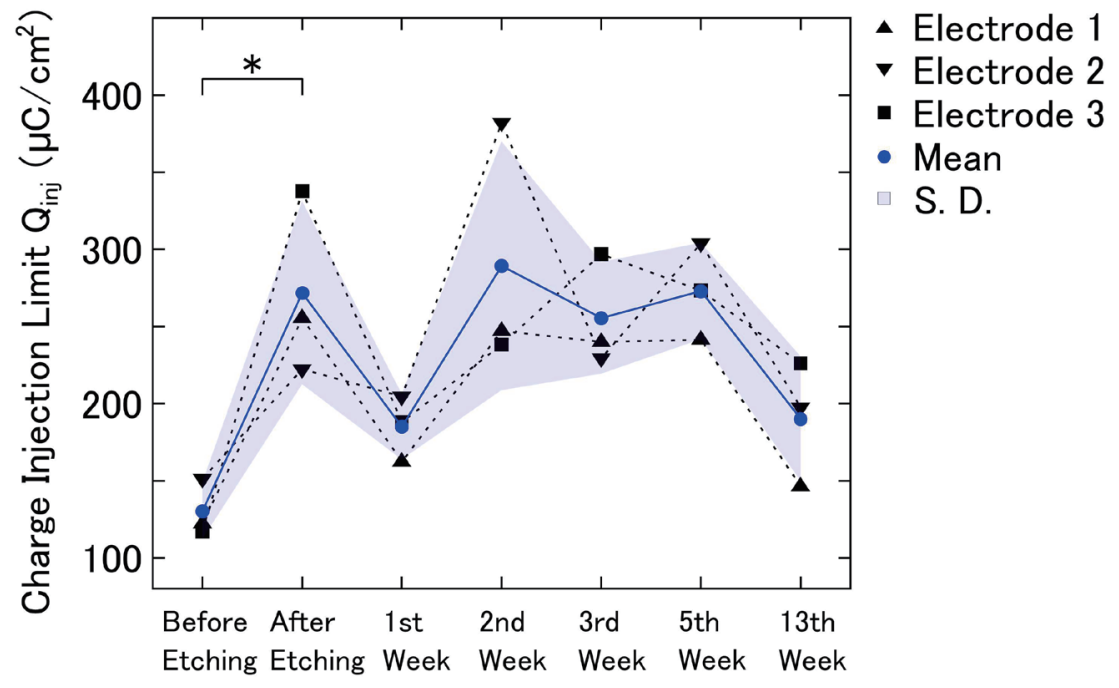

Fig. 7. (Color online) $Q_{i n j}$. The bar length and error bars represent mean and \pm S. D., respectively. The asterisk $(*)$ represents statistical significance $(p<0.05)$. The number of samples was three. 
holes were formed on the surface, and they were arranged in the same direction over a wide area of greater than $60 \mu \mathrm{m}$ [Fig. 3(B3)]. The cause of this long-range order is unknown, and further research is needed. Most of the crystal grains had an elongated shape (Fig. 4) presumably due to the use of a platinum wire that was stretched during processing. Regardless of the internal crystal structure, it is possible that the precipitation of salt caused the cube-like surface shown in Fig. 5. Further research on the mechanism of etching is necessary.

In the measurement of the cyclic voltammogram in Fig. 6(A), to confirm whether the potential window observed with the electrode used in this study does not differ greatly from the commonly used potential window ( -0.6 to $0.8 \mathrm{vs} \mathrm{Ag} / \mathrm{AgCl}$ ), a potential sweep over a wider range was performed. The current onset for oxygen generation was not significant even at a potential higher than $1 \mathrm{~V}$, but the increase in oxidation current could be observed from around $0.8 \mathrm{~V}$ by magnifying the graph [see Fig. 8(A)]. A similar less-significant current onset has also been reported in previous studies that reported a cyclic voltammogram with a wide sweep range. ${ }^{(20)}$ On the other hand, the current onset for hydrogen generation in the negative sweep was not significant even when it exceeded $-0.6 \mathrm{~V}$ [Fig. 8(B)], suggesting that the reduction current of dissolved oxygen was superimposed because nitrogen gas purge was not performed this time, making it difficult to see the current for hydrogen generation.

Two peaks were observed at 0.1 and $-0.2 \mathrm{~V}$ on the negative sweep of the voltammogram [Fig. 6(A)]. These seem to be peaks corresponding to the reduction reaction of platinum oxide, but the reason why they were observed separately was not clear. Two similar peaks were also reported in voltammetric studies, which measured platinum electrodes in PBS (e.g., Fig. 5 in Ref. 21 and Fig. 6 in Ref. 22, p. 198).

The CSCc of the electrode increased 2.4 times on average after etching, compared with before etching, but this was not statistically significant. This lack of statistical significance is considered to be due to a relatively large variation in the data (standard deviation) recorded after etching, compared with before etching, possibly due to individual differences in surface morphology after etching (Fig. 3). In this study, three bullet-shaped electrodes were used in the

\section{A}

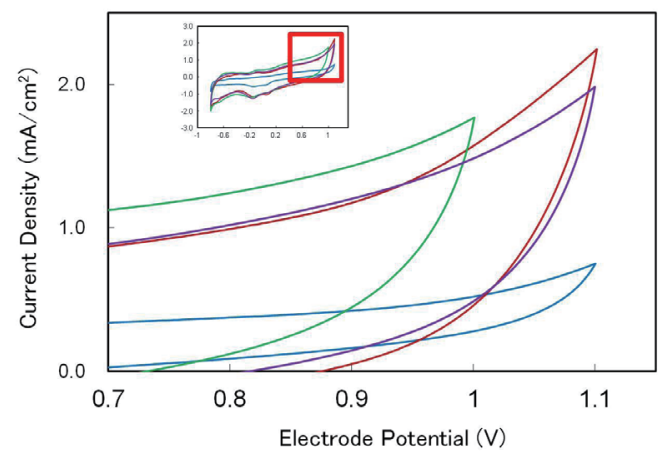

\section{B}

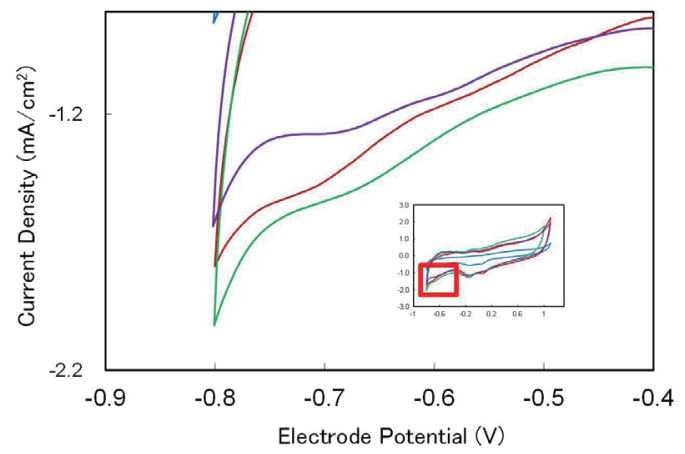

Fig. 8. (Color online) Magnification views of the cyclic voltammogram in Fig. 6(A). (A) Magnification of the positive end of the sweep range. (B) Magnification of the negative end of the sweep range. 
long-term stimulation study. This was due to the limitation of fabrication and experimentation; however, increasing the number of electrodes would help to detect statistical differences. Although this study was conducted using electrodes with a height of $0.5 \mathrm{~mm}$ as shown in Fig. 1, we have recently developed electrodes with a height of $0.3 \mathrm{~mm} .{ }^{(23)}$ Since the porosification of the electrode surface mainly occurred on the hemispherical portion of the bullet-shaped electrode and not so much on the sidewall of the electrode, a shorter electrode may result in a more significant increase in CSCc after anisotropic etching. Note that the increase in CSCc is not equivalent to the increase in $Q_{i n j}$. The inside of the pore of the porous electrode can be approximated by a distributed constant circuit composed of double-layer capacity and solution resistance ${ }^{(19)}$ and the time constant becomes larger toward the bottom of the pore. Accordingly, the deeper part of the pore is less utilized for charge transfer during electrical stimulation especially when the pulse width is small.

Pulses of current were continuously applied to the anisotropically etched electrodes for 13 weeks, but no significant change in surface morphology was observed. The amplitude of the pulse employed was $1.5 \mathrm{~mA}$, which is sufficiently greater than the maximum threshold of 1.0 $\mathrm{mA}$ reported in a clinical study describing a suprachoroidal retinal prosthesis. ${ }^{(18)}$ Therefore, the fact that no degradation was observed with the current intensity of $1.5 \mathrm{~mA}$ indicates that the anisotropically etched electrode has sufficient durability. In this study, the duration of continuous stimulation was limited to 13 weeks, and further research is required to investigate additional long-term durability. In the impedance spectrum, the value within the low-frequency region became slightly smaller at the 5th and 13th weeks, compared with that after etching [Fig. 6(C)]. A similar phenomenon has been reported in a previous study using different electrodes, ${ }^{(24)}$ suggesting that the cause of the impedance decrease in the low-frequency region was not the deterioration of the electrode but the deterioration of the insulation coating and the penetration of moisture into the metal insulation interface.

$Q_{i n j}$ was relatively low when tested after the 1st and 13th weeks of electrical stimulation (Fig. 7). This was suggested to be a measurement error because the variation of $Q_{i n j}$ was inconsistent with the results of CSCc, impedance, and surface morphology [Figs. 5, 6(B), and 6(D)]. In this study, the porous electrode was taken out from PBS used for continuous stimulation and was placed in air prior to electrochemical measurements; thus, the surface of the electrode may have dried. A possible explanation for the inconsistency of $Q_{i n j}$ and CSCc could be that, when the porous electrode with a dried surface was put in PBS, the air inside the small pores on the electrode surface remained adsorbed inside the pores, whereby the effective surface area of the electrode varied. This adsorption of the air may lower the electrode performance. This should be avoided by keeping the porous electrode always in wet state in a practical use.

In some cases, the entire electrode surface did not become uniformly porous after etching, but only a specific part of the surface became rougnened (Fig. 9). Even when the whole of the surface became porous, the surface morphology varied depending on the electrode (Fig. 3). The reason why the surface morphology differed between electrodes following etching is unclear. Further research such as the detailed morphological analysis of the electrode surface, the detailed analysis of the inner structure and crystal orientation of the electrode using crosssectional samples, and the identification of the bubble generation site during etching using a 

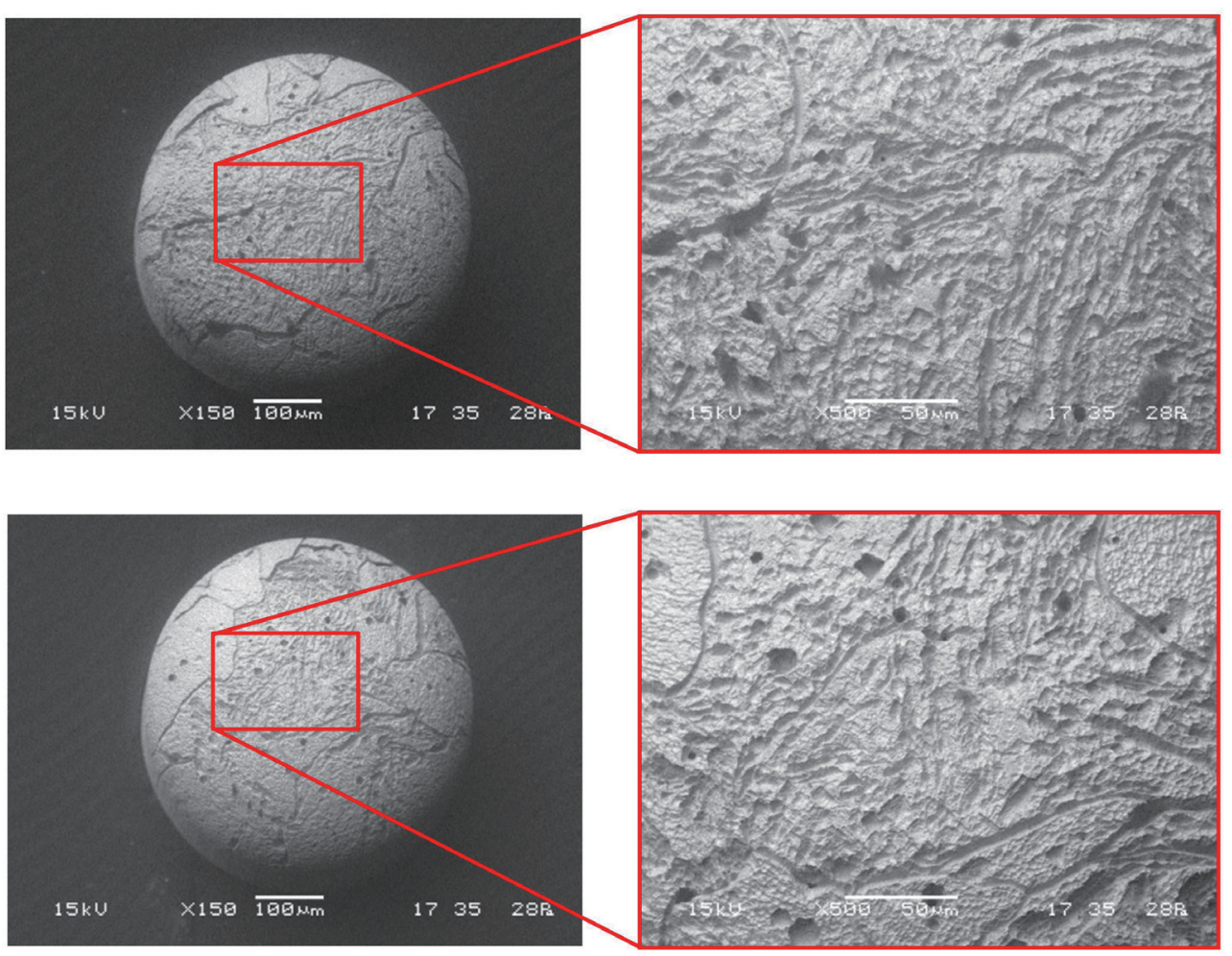

(A)

(B)

Fig. 9. (Color online) Two examples of etched electrodes that did not exhibit a uniform porous surface. Images in (B) are magnified views of (A).

high-speed camera at the electrode surface would help elucidate the cause of such differences revealed following anisotropic etching.

Table 1 shows a comparison of platinum surface treatment and charge injection coatings with respect to $Q_{i n j}$. $Q_{i n j}$ of 0.27 realized by anisotropic etching is not large compared with those of other methods. However, the advantage of the anisotropic etching is that the etching can be performed by a simple method that only periodically changes the polarity of the DC power supply in PBS. No special instruments or chemicals are required. Porosification by femtosecond laser irradiation recently reported by our group ${ }^{(23)}$ results in superior $Q_{i n j}$ and controllable hole size and density, but it takes long time to this laser processing because about 1000 holes are made in sequence for just one 500- $\mu \mathrm{m}$-diameter electrode. On the other hand, although this method requires $15 \mathrm{~h}$ for the etching process, multiple electrodes can be processed at the same time, so that it is advantageous when the number of electrodes to be processed is large. Although titanium nitride and iridium oxide are superior to anisotropic etching in $Q_{i n j}$, problems such as film delamination are unlikely to occur for an anisotropically etched electrode because it has no thin film. For porosification by other etching methods, Weremfo reported that the maximum $Q_{i n j}$ was $1 \mathrm{mC} / \mathrm{cm}^{2}{ }^{2(25)} Q_{i n j}$ was greatly dependent on the surface roughness and pulse width (Fig. 3.13 in Ref. 25). Although the $Q_{i n j}$ of the anisotropic etching proposed in this 
Table 1

Comparison of charge injection limits in different fabrication methods and materials.

\begin{tabular}{lccc}
\hline Material & Fabrication Technology & Maximun $Q_{i n j}\left(\mathrm{mC} / \mathrm{cm}^{2}\right)$ & Literature \\
\hline Platinum & Planar foil & $0.05-0.15$ & Rose et al. ${ }^{(4)}$ \\
Platinum & Laser irradiation & 0.1 & Green et al. ${ }^{(26)}$ \\
Platinum & Electrodeposition & 1 & Zhou et al..${ }^{(12)}$ \\
Platinum & Etching & 1 & Weremfo \\
Platinum & Laser irradiation & 0.8 & Terasawa et al. ${ }^{(23)}$ \\
Titanium nitride & Sputtering & 0.9 & Weiland et al..$^{(28)}$ \\
Iridium oxide & Sputtering & 5.75 & Weiland et al.. \\
PEDOT & Electrodeposition & 2.3 & Cui et al. ${ }^{(9)}$ \\
Platinum & Etching & 0.27 & This work \\
\hline
\end{tabular}

study is not relatively large, a higher $Q_{i n j}$ could be obtained by optimizing processing conditions and surface morphology. Note that all measurements including CV, impedance, and $Q_{i n j}$ were performed in air-equilibrated PBS in this study. The composition of dissolved gas in PBS could affect results of electrochemical measurements; thereby, care should be taken when comparing values such as $Q_{i n j}$ measured in air-equilibrated PBS with $Q_{i n j}$ measured in PBS equilibrated with other gases such as nitrogen.

\section{Conclusions}

In this paper, we have proposed a porosification method with anisotropic etching for platinum electrodes and evaluated the durability of the etched electrodes. The electrochemical properties of the electrodes were improved by anisotropic etching, and electrode durability was confirmed through a long-term stimulation study. In the future, we aim to elucidate the mechanism of the anisotropic etching phenomenon and perform further long-term durability studies.

\section{Acknowledgments}

We thank Yukari Nasu, Shiho Abe, Noriko Uchiyama, and Akihiro Uehara for performing etching experiments. We also thank Tohru Saitoh and Koji Oosawa for the fabrication of electrodes.

\section{References}

1 E. Greenbaum and D. Zhou, Eds.: Implantable Neural Prostheses 1: Devices and Applications (Springer-Verlag, New York, 2009). https://doi.org/10.1007/978-0-387-77261-5

2 L. da Cruz, B. F. Coley, J. Dorn, F. Merlini, E. Filley, P. Christopher, F. K. Chen, V. Wuyyuru, J. Sahel, P. Stanga, M. Humayun, R. J. Greenberg, G. Dagnelie, and Argus II Study Group: Br. J. Ophthalmol. 97 (2013) 632. https:// doi.org/10.1136/bjophthalmol-2012-30

3 E. Zrenner, K.-U. Bartz-Schmidt, C. Chee, F. Gekeler, T. L. Jackson, R. MacLaren, J. Nemeth, H. Sachs, K. Stingl, D. Wong, and S. S. Group: Invest. Ophthalmol. Vis. Sci. 55 (2014) E-abstract 5967.

4 T. L. Rose and L. S. Robblee: IEEE Trans. Biomed. Eng. 37 (1990) 1118. https://doi.org/10.1109/10.61038

5 S. B. Brummer and M. J. Turner: IEEE Trans. Biomed. Eng. 24 (1977) 440. 
6 M. Janders, U. Egert, M. Stelzle, and W. Nisch: Conf. Proc. IEEE Eng. Med. Biol. Soc. (IEEE, 1996) 1191.

7 X. Beebe and T. L. Rose: IEEE. Trans. Biomed. Eng. 35 (1988) 494. https://doi.org/10.1109/10.2122

8 S. F. Cogan, P. R. Troyk, J. Ehrlich, and T. D. Plante IEEE Trans. Biomed. Eng. 52 (2005) 1612. https://doi. org/10.1109/TBME.2005.851503

9 X. T. Cui and D. D. Zhou: IEEE Trans. Neural Sys. Rehabil. Eng. 15 (2007) 502. https://doi.org/10.1109/ TNSRE.2007.909811

10 S. Cogan: Proc. Neural Interfaces Conf. (NIC Orgainizers, 2010).

11 C. D. Lee, E. M. Hudak, J. J. Whalen, A. Petrossians, and J. D. Weiland: J. Electrochem. Soc. 165 (2018) G3015. https://doi.org/10.1149/2.0031812jes

12 S. F. Cogan, A. A. Guzelian, W. F. Agnew, T. G. H. Yuen, and D. B. McCreery: J. Neurosci. Meth. 137 (2004) 141. https://doi.org/10.1016/j.jneumeth.2004.02.019

13 M. Schuettler: Conf. Proc. IEEE Eng. Med. Biol. Soc. (IEEE, 2007) 186. https://doi.org/10.1109/ IEMBS.2007.4352254

14 C. Q. Huang and R. K. Shepherd: Hearing Res. 146 (2000) 57. https://doi.org/10.1016/S0378-5955(00)00100-3

15 D. D. Zhou, J. D. Dorn, and R. J. Greenberg: Conf. Proc. IEEE Multimedia Expo Workshops (IEEE, 2013$) 1$.

16 Y. Terasawa, H. Tashiro, K. Osawa, A. Yabusaki, M. Ozawa, T. Noda, and J. Ohta: Invest. Ophthalmol. Vis. Sci. 51 (2010) E-Abstract 3033.

17 Y. Terasawa, H. Tashiro, Y. Nakano, T. Tokuda, and J. Ohta: Adv. Biomed. Eng. 5 (2016) 137. https://doi. org/10.14326/abe.5.137

18 T. Fujikado, M. Kamei, H. Sakaguchi, H. Kanda, T. Endo, M. Hirota, T. Morimoto, K. Nishida, H. Kishima, Y. Terasawa, K. Oosawa, M. Ozawa, and K. Nishida: Invest. Ophthalmol. Vis. Sci. 57 (2016) 6147. https://doi. org/10.1167/iovs.16-20367

19 S. F. Cogan: Ann. Rev. Biomed. Eng. 10 (2008) 275. https://doi.org/10.1146/annurev.bioeng.10.061807.160518

20 E. M. Hudak, J. T. Mortimer, and H. B. Martin: J. Neural Eng. 7 (2010) 26005. https://doi.org/10.1088/17412560/7/2/026005

21 A. R. Harris, C. Newbold, P. Carter, R. Cowan, and G. G. Wallace: J. Neural Eng. 15 (2018) 046015. https:// doi.org/10.1088/1741-2552/aaba8b

22 D. Zhou and E. Greenbaum: Implantable Neural Prostheses 2: Techniques and Engineering Approaches (Springer-Verlag, New York, 2010). https://doi.org/10.1007/978-0-387-98120-8

23 Y. Terasawa, H. Tashiro, Y. Nakano, and J. Ohta: Sens. Mater. 30 (2018) 235. https://doi.org/10.18494/ SAM.2018.1652

24 S. R. Kane, S. F. Cogan, J. Ehrlich, T. D. Plante, and D. B. McCreery: Conf. Proc. IEEE Eng. Med. Biol. Soc. (IEEE, 2011) 5416.

25 A. Weremfo: Ph.D. Thesis of University of New South Wales (2015).

26 R. A. Green, H. Toor, C. Dodds, and N. H. Lovell: Sens. Mater. 24 (2012) 165. https://doi.org/10.18494/ SAM.2012.821

27 D. M. Zhou: US Patent No. US6974533B2 (2002).

28 J. D. Weiland, D. J. Anderson, and M. S. Humayun: IEEE. Trans. Biomed. Eng. 49 (2002) 1574. https://doi. org/10.1109/TBME.2002.805487 


\section{About the Authors}

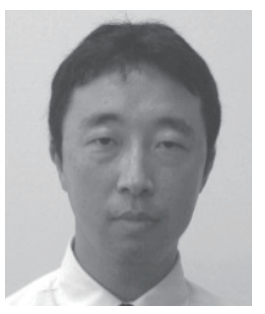

Yasuo Terasawa received his B.S. degree in applied physics and his M.S. degree in information science from Tohoku University, Miyagi, Japan in 1996 and 1998, respectively. He joined Nomura Research Institute in 1998. Since 2001, he has been an investigator in the Artificial Vision Institute of Nidek Co., Ltd., Aichi, Japan. He received his Ph.D. degree in materials science in 2009 from Nara Institute of Science and Technology (NAIST), Nara, Japan. Since 2009, he has been a research fellow in NAIST and an investigator in Nidek. Since 2016, he has been a manager of the Vision Institute in Nidek. His research interests include electrode technology, microfabrication, neural interfaces, and implantable electronics. He is a member of IEEE EMBS and the Association for Research in Vision and Ophthalmology.

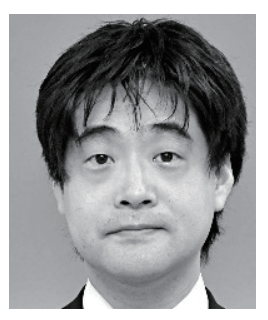

Hiroyuki Tashiro received his B.E. and M.E. degrees in electrical and electronic engineering from Toyohashi University of Technology (TUT), Aichi, Japan, in 1994 and 1996, respectively. He received his Ph.D. degree in engineering from Nara Institute of Science and Technology (NAIST), Nara, Japan, in 2017. In 1997, he worked as a clinical engineer at Kitasato University Hospital, Kanagawa, Japan. In 1998, he joined Nidek Co., Ltd., Aichi, Japan, where he worked on the research and development of ophthalmic surgical systems. He has been working on the development of retinal prosthetic devices since 2001. Since 2004, he has been an assistant professor in the Faculty of Medical Sciences, Kyushu University, Fukuoka, Japan. His research interests include biocompatibility and physiological evaluation of implantable electrical systems. He is a member of the Japanese Society of Medical and Biological Engineering, Japanese Society for Articial Organs, the Association for Research in Vision and Ophthalmology, and IEEE Engineering in Medicine and Biology Society.

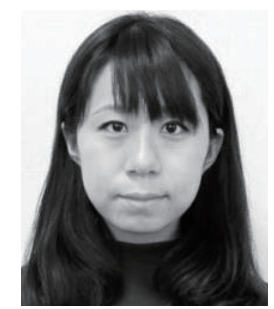

Yukari Nakano received her B.S. degree in chemical engineering from Shizuoka University, Shizuoka, Japan, in 2009. In 2009, she joined Nidek Co., Ltd., Aichi, Japan. Since 2012, she has been an investigator in the Artificial Vision Institute of Nidek Co., Ltd. She received her Ph.D. degree in materials science in 2018 from Nara Institute of Science and Technology (NAIST), Nara, Japan. Her research interests include the development of active implantable devices for blind people, electrophysiological evaluation, and the statistical analysis of electrophysiological evaluation. 


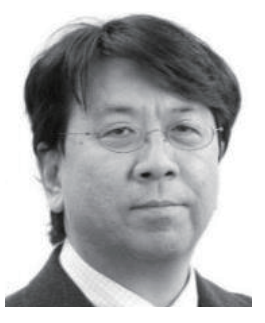

Jun Ohta received his B.E., M.E., and Dr. Eng. degrees in applied physics, all from the University of Tokyo, Japan, in 1981, 1983, and 1992, respectively. In 1983, he joined Mitsubishi Electric Corporation, Hyogo, Japan. From 1992 to 1993 , he was a visiting scientist at the Optoelectronics Computing Systems Center, University of Colorado at Boulder. In 1998, he joined the Graduate School of Materials Science, Nara Institute of Science and Technology (NAIST), Nara, Japan as an associate professor. He was appointed as a professor in 2004. His current research interests include smart CMOS image sensors for biomedical applications and retinal prosthetic devices. $\mathrm{He}$ received several awards including "The National Commendation for Invention" in 2001, "The Izuo Hayashi Award, Japanese Society of Applied Physics" in 2009, and so forth. He serves as a distinguished lecturer of IEEE SSCS, a section editor of IET Journal of Engineering, an associate editor of IEEE Trans. Biomedical Circuits and Systems, and so forth. He is a fellow of the Japan Society of Applied Physics and the Institute of Image, Information and Television Engineers and is a senior member of IEE Japan and IEEE. 